\title{
CATTANEO ILLUMINISTA
}

\author{
GIANNI FRANCIONI (*)
}

SunTo. - Attraverso il suo maestro Giandomenico Romagnosi, Carlo Cattaneo fece la conoscenza degli scritti di un autore decisivo per la sua formazione culturale: Giambattista Vico. La diffusione del pensiero del filosofo napoletano, peraltro già noto alla cultura lombarda almeno dagli anni Sessanta del XVIII secolo, aveva registrato un salto qualitativo con l'arrivo a Milano dei reduci della Repubblica partenopea, i quali avevano sottolineato gli elementi di vicinanza di Vico ai Lumi lombardi, a partire dal tema dell'incivilimento e delle leggi che lo regolano. Sulla scia di Romagnosi, Cattaneo dedicò al pensatore napoletano un interesse costante - maggiore di quello riservato a ogni altro filosofo, italiano e straniero - in tutta la sua produzione teorica. Pur nella consapevolezza dei limiti storici del pensiero di Vico - per certi aspetti in arretrato perfino rispetto ai suoi propri tempi - e quindi della distanza tra questo e l'illuminismo (in particolare lombardo) di cui rivendicava l'eredità, Cattaneo si sforzò quindi di svilupparne gli aspetti più fecondi.

$* * *$

ABstRaCT. - Carlo Cattaneo came to know Giambattista Vico's writings - that were to shape his education - through his master Giandomenico Romagnosi. The Neapolitan philosopher's thought had been known to Lombard intellectuals at least since the 1760s but it spread enormously when the Republicans fleeing Naples arrived in Milan and supported a reading of Vico side by side the Lombard Lumi, starting from the theme of civilized development and the laws supporting it. Following on from Romagnosi, Cattaneo devoted a steady interest - certainly stronger than any he had devoted to other philosophers, whether Italian or foreign - in the Neapolitan thinker through his whole theoretical production. Cattaneo strove to develop the most promising aspects of Vico's thought, even though he was aware of its historical limitations - that for certain aspects fell behind even his own times - and therefore of its distance from the Enlightenment, especially the Lombard Enlightenment, whose legacy he claimed to himself.

(*) Istituto Lombardo Accademia di Scienze e Lettere, Milano. Università di Pavia e Centro Linceo interdisciplinare "Beniamino Segre", Italia.

E-mail: gianni.francioni@unipv.it 
1. Sono stato tentato di intitolare questo intervento Cattaneo $e$ Vico (per le ragioni che si capiranno tra poco). Ho scelto infine lo stesso titolo del saggio premesso alla raccolta di Scritti filosofici, letterari e vari di Cattaneo, curata per i Classici italiani Sansoni nel 1957 da Franco Alessio, un maestro la cui memoria mi è cara ${ }^{1}$. Quell'edizione era comparsa in un momento in cui si era riaperta la disputa storiografica sulla collocazione di Cattaneo entro le diverse correnti filosofiche dell'età moderna. Tredici anni più tardi, affrontando - in un saggio poi raccolto nel volume Una filosofia militante. Studi su Carlo Cattaneo - un quesito divenuto un vero topos della critica («illuminista o positivista?»), Norberto Bobbio passerà in rassegna le interpretazioni che si erano succedute a partire dal 1877, quando Alberto Mario, uno dei primi studiosi del pensatore lombardo, aveva accostato gli studi cattaneani sulla psicologia delle menti associate a quelli del capofila del positivismo italiano, Roberto Ardigò, sulla Psicologia come scienza positiva (è il titolo di un'opera di Ardigò, uscita peraltro nel 1870; Cattaneo era morto l'anno prima) $)^{2}$. Nel 1878 Enrico Zanoni aveva indicato in Romagnosi e Cattaneo «le due avanguardie più poderose del positivismo italiano» ${ }^{3}$. Col nuovo secolo, in piena reazione neo-idealistica al positivismo, sarà Giovanni Gentile ad accreditare autorevolmente Cattaneo come «primo positivista italiano» ${ }^{4}$ (e va da sé che, mentre per i seguaci tardoottocenteschi di Cattaneo il termine positivista era da intendere come un complimento, «detto da Gentile, nel momento in cui ferveva la battaglia per liberare l'Italia dalla muffa positivistica, [era] quasi un insulto» ${ }^{5}$. Gentile, oltretutto, negava a Cattaneo la qualifica di filosofo).

Una contrapposta definizione di Cattaneo come «l'ultimo degli illuministi» $»^{6}$ si era invece fatta strada nella cultura italiana del secondo

1 F. Alessio, Cattaneo illuminista, introduzione a C. Cattaneo, Scritti filosofici, letterari e vari, Firenze, Sansoni, 1957.

2 A. Mario, Teste e figure. Studi biografici, Padova, Salmin, 1877, 416, cit. in N. Bobbio, Una filosofia militante. Studi su Carlo Cattaneo, Torino, Einaudi, 1971.

3 E. Zanoni, Carlo Cattaneo, Milano, Tipografia Gattinoni, 1878; seconda edizione accresciuta: Carlo Cattaneo nella vita e nelle opere, Roma, Società editrice Dante Alighieri, 1898, 334

4 G. Gentile, Le origini della filosofia contemporanea in Italia, II, I positivisti, Messina, Principato, 1921, 27.

5 Bobbio, Una filosofia militante, cit., 125.

6 Ivi, 127. 
dopoguerra (entro la quale si era sviluppato per un certo periodo un indirizzo di pensiero anti-idealistico chiamato "neoilluminismo" ${ }^{7}$. Nel saggio citato, Alessio parlava non a caso della posizione filosofica di Cattaneo come «caratterizzata da uno strenuo e costruttivo richiamo illuministico alla determinatezza dei problemi ed alla efficacia di soluzioni effettive» ${ }^{8}$. E sulla stessa lunghezza d'onda si collocavano in quegli anni altri interpreti, come Sergio Romagnoli, per il quale delineare «una stretta, consequenziale parentela Cattaneo-positivismo» era solo il frutto di un «equivoco»?

Le categorie storiografiche non sono «strumenti di precisione», osservava ancora Bobbio, «e gli strumenti non raffinati, si sa, producono effetti diversi secondo la mano che li usa» ${ }^{10}$. Gli ismi (come li chiamava efficacemente un altro maestro pavese, Giulio Preti) sono solo schemi utili per sintetizzare i risultati di ricerche concrete. Dare a Cattaneo dell'illuminista o del positivista, come volta a volta si è continuato a fare, dipende in definitiva dalle componenti teoriche su cui si vuol far cadere l'accento, cioè dai tratti che lo storico intende sottolineare perché li ritiene predominanti nel suo pensiero. Bisogna tuttavia riconoscere che, nel caso di Cattaneo, le cose risultano complicate dall'essersi egli trovato «in un'età di transizione» ${ }^{11}$ tra le estreme riproposizioni del riformismo illuministico lombardo e il pieno affermarsi della cultura positivistica dell'ultimo scorcio dell'Ottocento. Una funzione di transizione di cui peraltro lo stesso Cattaneo ebbe consapevolezza, se in una lettera del 1854, riflettendo sulla propria operosa attività di pensatore, poteva scrivere di aver avuto «il debito d'una tradizione da conservare al mio paese e forse nessun miglior modo di riempire un intervallo» ${ }^{12}$.

7 Cfr. in proposito Il neoilluminismo italiano. Cronache di filosofia (19531962), a cura di M. Pasini e D. Rolando, Milano, il Saggiatore, 1991; Massimo Mori, Il neoilluminismo italiano, in Il contributo italiano alla storia del pensiero - Filosofia, Roma, Istituto della Enciclopedia Italiana, 2012 (consultato on line).

8 Alessio, Cattaneo illuminista, cit., XVIII.

9 S. Romagnoli, Carlo Cattaneo tra illuminismo e positivismo, in Rivista storica del socialismo, I, 1958, n. 3, 244.

10 Bobbio, Una filosofia militante, cit., 128.

11 Ibidem.

12 Epistolario di Carlo Cattaneo, raccolto e annotato da R. Caddeo, 4 voll., Firenze, Barbèra, 1949-56, II, 313. 
Fatte queste precisazioni, Bobbio non aveva dubbi, nel suo libro del 1971, sul fatto che Cattaneo potesse essere sì definito «scienziato positivo», ma non positivista in senso proprio; mentre, per contro, gli pareva innegabile che la sua cultura si riallacciasse, «attraverso Romagnosi suo maestro, ai riformatori lombardi, dai Verri al Carli, al Beccaria», coi quali condivideva «la incondizionata fiducia nella potenza rischiaratrice e civilizzatrice dell'intelligenza che squarcia le tenebre dell'ignoranza e della superstizione e distrugge a poco a poco la barbarie dell'uomo primitivo», e ancora «la concezione ottimistica dell'uomo e della storia» e l'entusiasmo per ogni scoperta scientifica o innovazione tecnologica (seguite, potremmo dire, con un'attenzione "baconiana", in quanto strumenti per il miglioramento dell'umanità; il che è ovviamente cosa ben diversa dal culto positivistico della scienza). Questi tratti si coniugavano in Cattaneo alla convinzione che «progresso tecnico e progresso civile dovessero procedere di pari passo», del resto confermata dal suo «radicato atteggiamento riformistico» nei confronti delle istituzioni esistenti ${ }^{13}$.

Per quel che più ci interessa in questa sede, la distinzione che Cattaneo operava tra la «filosofia delle scuole» e quella che chiamava la «filosofia popolare» (dove con "popolo" intendeva riferirsi alla classe borghese che si veniva formando nel paese) ${ }^{14}$ rimandava ad analoghe prese di posizione degli illuministi lombardi (più volte espresse ne «Il Caffè»); la «filosofia popolare» finiva poi per coincidere con la «filosofia scientifica» (o, come la chiamava talvolta, con la «filosofia esperimentale» ${ }^{15}$, fondata cioè sullo studio dei fatti).

Si tratta comunque, per Cattaneo, di un sapere pubblico, utile alla comunità degli uomini, e dunque alieno da ogni pensiero astratto, contemplativo, riservato a conventicole di iniziati e non finalizzato al progresso e al benessere della società. Dunque, in senso proprio, una «filosofia militante» (l'espressione è pressoché testuale in Cattaneo: «la filosofia è una milizia» $)^{16}$, che non a caso preferisce adottare una prosa

13 Bobbio, Una filosofia militante, cit., 5-7, passim.

14 Cfr. ivi, 98.

15 Cfr. ivi, 102.

16 Con questa affermazione, come sottolineato da Bobbio, Cattaneo aveva concluso le sue lezioni luganesi di Logica (cfr. C. Cattaneo, Scritti filosofici, a cura di N. Bobbio, 3 voll., Firenze, Le Monnier, 1960, III, 329). 
piana e priva di orpelli retorici (a parte quella sua dannata mania di mettere accenti ovunque...), che ama esprimersi nella forma del saggio breve piuttosto che del trattato, nella pubblicazione periodica piuttosto che nella monografia, e che, rifiutando ogni forma di pensiero speculativo, rivela, anche in questo, una concezione dell'attività intellettuale come fatto squisitamente sociale e politico.

Cattaneo usava spesso un'altra formula, «filosofia civile», che riprendeva direttamente da Romagnosi. Per il giurista di Salsomaggiore, la «civile filosofia» consisteva in un sapere capace di dare unità di scienza a tutto ciò che concerne l'uomo singolo e l'uomo associato, che ha per fine la felicità individuale e quella collettiva. Teoria della conoscenza e psicologia, filosofia della storia, morale e scienza della legislazione venivano a connettersi nel suo pensiero in un'idea di filosofia che da una parte mostrava un suo marcato orientamento pratico-operativo e antimetafisico di matrice illuministica, dall'altra recuperava l'ideale umanistico di un sapere al servizio della civitas. Lo studio dell'incivilimento (e il termine aveva di per sé un'ascendenza illuministica) ne costituiva il campo d'indagine privilegiato. Incivilimento è la possibilità di organizzare e guidare quella capacità produttiva che è rappresentata dalla perfettibilità dell'uomo, in un processo che non è infinito, ma trova il proprio compimento quando lo Stato raggiunge la sua «potenza civile», nell'equilibrio dei suoi componenti e nel massimo grado di sviluppo delle conoscenze e dei lumi. Romagnosi aveva attribuito a Giambattista Vico il merito di aver fondato per primo in Italia la «filosofia civile», di aver compreso che lo sviluppo umano è governato da leggi precise.

Fedele all'insegnamento romagnosiano, Cattaneo pone al centro della sua riflessione il grande tema illuministico del progresso, nel quale vengono ad annodarsi le sue analisi sulle lingue, i costumi, le istituzioni, i mutamenti sociali presso i diversi popoli, delineando così il quadro di una filosofia della storia capace di individuare le leggi di tendenza che presiedono alle vicende delle nazioni (leggi che tuttavia, a differenza di quelle "scoperte" di lì a poco dai positivisti à la Comte, nulla hanno di aprioristico e di deterministico ${ }^{17}$. In questa prospettiva, probabilmente all'inizio degli anni Venti (quando comincia a seguire le lezioni private di Romagnosi), Cattaneo studia la Scienza nuova di Vico, che in seguito riconoscerà essere stata decisiva nella sua formazione culturale.

17 Cfr. Bobbio, Una filosofia militante, cit., 112. 
2. Apriamo una parentesi. Quando Cattaneo affronta lo studio della Scienza nuova, la diffusione del pensiero di Vico nella cultura lombarda è un fatto ormai consolidato ${ }^{18}$. La storiografia neo-idealistica (Benedetto Croce in primo luogo) ha a lungo sostenuto che Vico era rimasto sconosciuto in Lombardia fino all'alba del XIX secolo, quando Milano, grazie alla propaganda degli esuli della Repubblica partenopea del 1799, era diventata «la seconda roccaforte italiana del vichismo». Solo quell'innesto di idee, e solo a far tempo da quell'evento, aveva provocato mutamenti profondi in una filosofia fino a quel momento caratterizzata dalla sua dipendenza dalle lumières francesi e dalla carenza di senso della storia ${ }^{19}$. La critica ha in seguito confutato questo schema interpretativo, dimostrando che Vico è un autore noto e apprezzato (anche se forse più citato che studiato a fondo) ben prima dell'arrivo dei patrioti napoletani, addirittura - come è stato messo in luce da recenti contributi - già nel periodo di formazione di quella che Voltaire chiamò l'école de Milan: negli anni, cioè, del Dei delitti e delle pene; sicché il successivo diffondersi nella cultura milanese di teorie del progresso e dell'incivilimento di chiara ascendenza vichiana poté innestarsi su un filone di pensiero radicato e orientato.

La circolazione delle idee del filosofo napoletano nel Settentrione era partita dal Veneto, dove Vico, ancora in vita, aveva avuto una sua area di diffusione editoriale e dei conoscitori attenti, come Apostolo Zeno e Antonio Conti, e aveva toccato Milano alla metà degli anni Sessanta del XVIII secolo. Vico, «grande e stranissimo autore», era stato ricordato ne «Il Caffè» per aver sostenuto «che Omero non vi sia stato, e che altro non sieno i suoi poemi che una collezione di antichi canti, come ne abbiamo un esempio in Ossian ${ }^{20}$. Poi la lezione vichiana aveva ispirato diversi passaggi del Saggio sulla storia d'Italia di

18 Su questo tema cfr., anche per la bibliografia, il mio Una filosofia civile. Contributo alla storia dell'Università di Pavia tra la fine del Settecento e gli inizi dell'Ottocento, in Università degli Studi di Pavia - 12 dicembre 2002 - Inaugurazione dell'Anno Accademico 2002-2003, Pavia, 2003, 55-74, che ho in parte ripreso nel seguito. Si veda inoltre G. Cospito, Il «gran Vico». Presenza, immagini e suggestioni vichiane nei testi della cultura italiana pre-risorgimentale (1799-1839), Genova, Name, 2002.

19 Cfr. B. Croce, Bibliografia vichiana, accresciuta e rielaborata da F. Nicolini, 2 voll., Napoli, Ricciardi, 1947, 407 sgg.

20 A. Verri, Dei difetti della letteratura e di alcune loro cagioni, in Il Caffè (17641766), a cura di G. Francioni e S. Romagnoli, Torino, Bollati Boringhieri, $1998^{2}$, 542. 
Alessandro Verri, dove Vico era stato definito «un grand'uomo, il di cui nome non è forse così celebre quanto meriterebbe»: egli aveva inteso ricostruire «la vita del genere umano», spiegare cioè «come l'uomo si unisse in società, e come in lui nascessero le idee religiose e morali, quai sieno i princìpi della legislazione, per quai gradi progrediscano le nazioni alla coltura, per quali ritornino nella barbarie. [...] Non so - concludeva Verri - se l'Italia abbia avuto un più gran pensatore ${ }^{21}$. Né si trattava di un caso isolato, se si pensi all'ultimo capitolo delle Ricerche intorno alla natura dello stile di Cesare Beccaria (rimasto inedito fino al 1809 ma scritto intorno al 1770), dove il progresso delle nazioni era visto vichianamente come necessario passaggio da uno «stato selvaggio e primitivo» (quando le nazioni sono «abbandonate alla naturale loro perfettibilità ed al lento sviluppamento delle loro facoltà»), ad uno «stato poetico, imaginoso ed eloquente», a un terzo e ultimo stadio, quello che si manifesta «al giorno d'oggi presso le colte nazioni» ${ }^{22}$.

Con gli anni Ottanta, un più diretto contatto fra la cultura napoletana e quella lombarda era stato favorito da Aurelio De Giorgi Bertòla, professore di Storia universale all'Università di Pavia dal 1784, dopo un decennio trascorso a Napoli in dimestichezza con esponenti della seconda generazione vichiana, fra i quali Mario Pagano, autore di un'opera - i Saggi politici, usciti in prima edizione nel 1783-85 - che rappresentava la più elaborata ripresa del pensiero di Vico nella cultura illuministica meridionale e costituiva un interessante tentativo di coniugare l'eredità vichiana coi Lumi europei. Bertòla aveva pubblicato a Pavia nel 1787 un libro intitolato Filosofia della storia, che aveva avuto vasta diffusione, anche all'estero. Con questa espressione, che veniva usata in Italia per la prima volta, Bertòla intendeva riferirsi ad un tipo di analisi storica che, indagando «le molte vedute, le molte relazioni, i molti influssi, le combinazioni molte» e mettendo in luce «i varj e sottili aspetti delle analogie e delle differenze», fosse in grado di cogliere «i principj, i progressi, l'incatenamento, le conseguenze delle azioni

21 A. Verri, Saggio sulla storia d'Italia, a cura di B. Scalvini, Roma, Edizioni di Storia e Letteratura, 2001, 317-18.

22 Ricerche intorno alla natura dello stile, in C. Beccaria, Scritti filosofici e letterari, a cura di L. Firpo, G. Francioni e G. Gaspari, Milano, Mediobanca, 1984 (Edizione Nazionale delle opere di Cesare Beccaria, diretta da L. Firpo e G. Francioni, II), 71, 73, 189, 193, 194, 198. 
umane». Difficile non pensare a Vico; come è difficile non riportare alla Scienza nuova un altro tema presente nella Filosofia della storia, quello dell'immaginazione poetica espressa nelle favole e nei miti, che a parere di Bertòla dovevano essere assunti come materiale per la ricostruzione di una «storia filosofica degli uomini» ${ }^{23}$. E per restare a Pavia, esemplare della crescente attenzione per le idee di Vico è il caso del futuro giacobino Pietro Custodi, alunno del Collegio Borromeo dal 1790 al 1795, così interessato al filosofo napoletano da arrivare, pur di possedere la quasi irreperibile Scienza nuova, a ricopiarla interamente a mano con l'aiuto di un compagno di studi.

Intanto, negli anni a cavallo fra Sette e Ottocento, l'interesse per la storia si allargava in Lombardia ad una riflessione sull'incivilimento, il progresso, la perfettibilità, che da una parte riecheggiava una tematica centrale nella filosofia francese di quel periodo (si pensi a Condorcet), dall'altra riprendeva analoghe discussioni già presenti nella cultura lombarda (il termine «perfettibilità», ad esempio, è già attestato in Beccaria e nelle pagine de Il Caffè): un dibattito destinato a durare a lungo e a intersecarsi con la riflessione di Romagnosi.

Il terreno dunque è già dissodato quando, nel 1800, un nutrito gruppo di esuli della Repubblica partenopea (da Vincenzo Cuoco a Francesco Lomonaco, da Francesco Salfi a Flaminio Massa) si rifugia a Milano e vi comincia una martellante propaganda, nella quale si distingue in particolare Cuoco, che nei suoi scritti giornalistici si fa propagatore delle idee vichiane, ma anche assertore di una continuità tra l'illuminismo napoletano (visto come erede del pensiero di Vico) e i Lumi lombardi di Verri e Beccaria. Nel 1801 ha inizio a Milano anche un'attività editoriale destinata a continuare nei decenni successivi: esce la prima ristampa in assoluto della Scienza nuova (per probabile iniziativa di Vincenzo Monti); nello stesso anno viene approntata - quasi sicuramente da Pietro Custodi - la versione italiana di un'altra opera di Vico, il De antiquissima Italorum sapientia (ma verrà pubblicata solo nel 1816 dal tipografo Silvestri, che dichiarerà di aver ricevuto il manoscritto dell'originale da Romagnosi). Più avanti, tra il 1835 e il 1837, sarà un giovane seguace di Romagnosi, Giuseppe Ferrari, a curare la prima raccolta delle opere complete di Vico, in 6 volumi.

23 A. De' Giorgi Bertòla, Della filosofia della storia, a cura di F. Lomonaco, Napoli, Liguori, 2002, 5 e passim. 
A partire dall'inizio dell'Ottocento, è possibile registrare una crescente presenza di Vico nel pensiero di vari autori (citerò solo i primi): da Francesco Lomonaco (un esule che trascorrerà qui il resto della sua vita, e che "inizierà" al vichismo molti lombardi, a partire dal giovane Alessandro Manzoni), a Vincenzo Monti e Ugo Foscolo (docenti di eloquenza a Pavia, rispettivamente, nel 1802-1804 e nel 1809) a Giandomenico Romagnosi, appunto (che pure non aveva atteso gli esuli napoletani per scoprire Vico, risalendo la sua lettura della Scienza nuova all'inizio degli anni Ottanta del Settecento, quando era ancora studente nel Collegio Alberoni di Parma).

Nella sua prolusione pavese del 1808, sull'Origine e i progressi della civile giurisprudenza, Romagnosi elogiava Vico ed esponeva gli esiti di una sua riflessione cominciata nel decennio precedente, che riconduceva tutti i fenomeni (psicologici, morali, politici ecc.) che compongono il «costume naturale delle nazioni» a «leggi costanti e generali di fatto, che non variarono, che non variano né varieranno giammai, poste o ripetute simili situazioni», e si soffermava sul tema del progresso e sui modi di «incessantemente innoltrare l'incivilimento fino al punto che la nazione rendasi sicura e felice», ponendo in primo piano l'esigenza di individuare «una legge certa e graduale derivante dalla natura dell'uomo e dall'ordine delle cose eterne», una «legge unica e necessaria che promuove e sospinge il perfezionamento morale e politico delle civili società $\rangle^{24}$. E se, descrivendo in uno scritto del 1796 la lenta evoluzione delle società, Romagnosi aveva ripreso pressoché alla lettera Vico per scandire, nel processo dell'incivilimento, tre epoche, ovvero «l'età dei sensi, l'età dell'immaginazione e l'età della ragione» ${ }^{25}$, nelle Osservazioni su la Scienza Nuova di Vico del 1822 non aveva mancato di esprimere le sue riserve su una serie di aspetti non secondari del pensiero del napoletano: dall'assenza di una precisa teoria dei sentimenti religiosi all'uso spregiudicato delle antiche mitologie, alla descrizione dello stato primitivo della specie umana. Riserve che non gli avevano però impedito di costellare di citazioni e di rimandi a Vico anche una delle sue ultime opere (forse il suo capolavoro), il saggio del 1832 Dell'indole e dei fattori dell'incivilimento, dove aderiva

24 Origine e progressi della civile giurisprudenza, in G.D. Romagnosi, Opere edite e inedite, a cura di A. De Giorgi, 8 voll., Milano, Perelli e Mariani - Volpato, 184148, II, 371, 372.

25 Romagnosi, Lettere al Signor Gian Piero Vieusseux, ivi, III, 528. 
ancora una volta alla visione stadiale del progresso dell'umanità che Vico aveva codificato nella Scienza nuova.

3. Chiudiamo questa lunga parentesi. Ci è servita a delineare il retroterra che spiega l'ininterrotto interesse di Cattaneo per $\mathrm{Vico}^{26}$. A partire dal saggio Su la Scienza Nuova di Vico (uscito nel 1839 sul Politecnico e riproposto nel 1846 in una versione in più punti modificata), il filosofo napoletano rimarrà una presenza costante nei suoi scritti teorici più importanti, dalle prefazioni ai sette volumi della prima serie del Politecnico (1839-44) alle lezioni sulla Psicologia delle menti associate tenute all'Istituto Lombardo tra il 1859 e il 1866, il cui testo critico è finalmente disponibile nell'edizione attentamente curata da Barbara Boneschi. Non è un caso se, nell'indice dei nomi dell'Edizione nazionale delle opere cattaneane, quello di Vico sia, tra i filosofi, il più citato. Il grande merito di Vico era stato, per Cattaneo, non soltanto di aver applicato il metodo sperimentale alle scienze umane, e in particolare a quelle storico-sociali, ma specialmente di essere andato oltre Locke, che si era limitato all'esame dell'individuo astrattamente considerato, e di aver così inaugurato «lo studio dell'individuo nel seno dell'umanità, l' $i$ deologia sociale» ${ }^{27}$, cioè lo studio dello sviluppo della mente umana nella società e nella storia.

Lettore non acritico, anche Cattaneo non mancava di marcare i propri punti di dissenso rispetto al filosofo napoletano: gli rimproverava tra l'altro di essere ricorso, per «risolvere il problema dell'improvviso trapasso dei primitivi selvaggi dall'errare ferino alla vita agricola», alla «imaginaria ipotesi del primo fulmine e dell'improvviso culto di Giove Tonante» ${ }^{28}$, e di aver ipostatizzato la storia greca e specialmente quella romana a modello di sviluppo dell'umanità tutta. Ma in particolare, pur riconoscendogli il merito di aver fondato «una scienza di tutte

26 Sul tema cfr. Cospito, Il «gran Vico», cit., parte I, Il Vico di Cattaneo, 1365. Le pagine dedicate da Cattaneo a Vico sono utilmente raccolte in C. Cattaneo, $\mathrm{La}$ scienza nuova dell'umanità. Scritti vichiani 1836-1861, a cura di G. Cospito, Genova, Name, 2002.

27 Su la Scienza Nuova di Vico, in Cattaneo, La scienza nuova dell'umanità, cit., 50 .

28 C. Cattaneo, Psicologia delle menti associate. Le "letture" di Carlo Cattaneo all'Istituto Lombardo di Scienze e Lettere, edizione critica a cura di B. Boneschi, Milano, Istituto Lombardo di Scienze e Lettere, 2016, 120. 
le istorie, una legge universale che guidi tutti i popoli, un'istoria ideale eterna commune a tutte le nazioni ${ }^{29}$, gli rimproverava (ed era una critica già mossagli da Romagnosi) di non essere riuscito a fornire un'idea adeguata di progresso. Cattaneo, come ha scritto Carlo Lacaita nel bel saggio introduttivo all'edizione della Psicologia delle menti associate che oggi festeggiamo, «aveva rivisto l'illuministica idea del progresso indefinito e lineare» approdando a una «rappresentazione del cammino umano nella storia come il corso irregolare di un fiume segnato da accelerazioni, stagnazioni e arretramenti, ma pur sempre in movimento ${ }^{30}$ (come si legge nella Psicologia delle menti associate, «vi è nelle nazioni il progresso, ma v'è anche il regresso e il decadimento [...]. Il progresso prevale perché col corso del tempo cresce naturalmente il numero delle verità») $)^{31}$. Per Cattaneo, il limite di Vico era di essersi invece fermato al «circolo», alla «ruota fatale» della storia ${ }^{32}$, cioè ad una visione ciclica, mutuata da Campanella e Machiavelli.

Poco importa - sia detto per inciso - rilevare qui quanto questa interpretazione fosse errata, come sarà errata quella, ben più nota, successivamente avanzata da Benedetto Croce, il primo a parlare, a proposito di Vico, di «corsi» e «ricorsi», al plurale ${ }^{33}$; laddove in Vico il termine figura sempre al singolare, e il campo di applicazione di questo concetto è limitato ad un solo caso storico, quello della «barbarie ritornata» del Medioevo, né mai si accompagna all'idea che nella «storia ideale eterna» un «ricorso» debba necessariamente avvenire. Vico cioè non fa del «ricorso» la componente essenziale di un modello ciclico della storia, e meno che mai pensa che possa darsi un «ricorso» dello stadio dell'erramento ferino ${ }^{34}$. Dunque Romagnosi e Cattaneo, a questo proposito, si sbagliavano; ma una lunga schiera di interpreti ha fatto loro compagnia, fino a tempi recenti.

29 Cattaneo, Su la Scienza Nuova di Vico, cit., 60.

30 C.G. Lacaita, Cattaneo filosofo moderno, in Cattaneo, Psicologia delle menti associate, cit., 42 .

31 Cattaneo, Psicologia delle menti associate, cit., 149.

32 Epistolario di Carlo Cattaneo, cit., IV, 504.

33 B. Croce, La filosofia di Giambattista Vico, Bari, Laterza, $1933^{3}$, in particolare 127.

34 Cfr. P. Cristofolini, La "Scienza nuova" di Vico. Introduzione alla lettura, Roma, La Nuova Italia Scientifica, 1995, 139-42. 
4. Mi fermo, per ragioni di tempo, a queste poche e marginali osservazioni. Ma non prima di aver precisato che Cattaneo non assimilava (né l'aveva fatto Romagnosi) il pensiero di Vico all'illuminismo, e affermava anzi con chiarezza che il filosofo napoletano «ricusava pensare col suo secolo» ${ }^{35}$. Oggi la storiografia ha ampiamente indagato la compresenza in Vico di geniali intuizioni e di attardamenti barocchi, frutto di un continuo dialogo con autori del passato, che ignora i contemporanei. Il Vico illuminista era un'invenzione dei suoi seguaci meridionali, nel cui progetto politico-culturale era essenziale dipingerlo come pensatore pienamente al passo col proprio tempo. Cattaneo non aveva alcun interesse a percorrere quella strada. Ma innestando nel suo patrimonio culturale di innegabile marca illuministica le idee di Vico, egli mostrava di considerare la filosofia dei Lumi non come un'eredità da conservare con ossequio, e da riproporre in formule vuote o superficiali, ma come un corpo vivo, suscettibile di incrementi teorici innovativi.

35 Cattaneo, Su la Scienza Nuova di Vico, cit., 63. 